International Journal of Pure and Applied Mathematics

Volume 104 No. 4 2015, 599-619

ISSN: 1311-8080 (printed version); ISSN: 1314-3395 (on-line version)

url: http://www.ijpam.eu

doi: http://dx.doi.org/10.12732/ijpam.v104i4.11

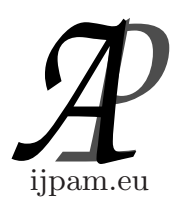

\title{
GENERALIZATION OF $m$-PARTIAL ISOMETRIES ON A HILBERT SPACE
}

\author{
Ould Ahmed Mahmoud Sid Ahmed \\ Department of Mathematics \\ College of Science \\ Aljouf University \\ Al Jouf, 2014, SAUDI ARABIA
}

\begin{abstract}
In this paper we introduce a generalization of the class of $m$-partial isometries operators recently studied in [24]. A bounded linear operator $T$ on a Hilbert space $\mathcal{H}$ is called an $m$ - partial isometry of order $q$ for a positive integers $m$ and $q$, if

$$
T^{q}\left(T^{* m} T^{m}-\left(\begin{array}{c}
m \\
1
\end{array}\right) T^{* m-1} T^{m-1}+\left(\begin{array}{c}
m \\
2
\end{array}\right) T^{* m-2} T^{m-2}-\cdots+(-1)^{m} I\right)=0 .
$$
\end{abstract}

\section{AMS Subject Classification: 47B48}

Key Words: Hilbert space, isometry, partial isometry, $m$-isometric operator, reducing subspace, the single valued extension property

\section{Introduction and Preliminaries Results}

Let $\mathcal{H}$ denotes on a complex a separable infinite dimensional Hilbert space and $\mathcal{B}(\mathcal{H})$ the algebra of bounded linear operators on $\mathcal{H}$ into itself. For $T \in \mathcal{B}(\mathcal{H}), T^{*}$ denotes the adjoint of $T, \mathcal{R}(T)$ and $\mathcal{N}(T)$ denote the range and the null-space of $T$, respectively, $I=I_{\mathcal{H}}$ being the identity operator.

Received: August 8, 2015

(c) 2015 Academic Publications, Ltd. url: www.acadpubl.eu 
One of the most important subclasses, of the algebra of all bounded linear operators acting on a Hilbert space, the class of partial isometries operators. The operator theory of partial isometries has been studied by several authors ([12], [18]).

An operator $T \in \mathcal{B}(\mathcal{H})$ is said to be self-adjoint if $T^{*}=T$, isometry if $T^{*} T=I$ and partial isometry if $T T^{*} T=T$. In recent years this classes has been generalized, in some sense, to the larger sets of operators so-called $m$-selfadjoint, $m$-isometry and $m$-partial isometry.

An operator $T \in \mathcal{B}(\mathcal{H})$ is called $m$-self- adjoint for some integer $m \geq 1$ if

$$
\sum_{0 \leq k \leq m}(-1)^{k}\left(\begin{array}{c}
m \\
k
\end{array}\right) T^{* k} T^{m-k}=0
$$

and it is called $m$-isometry for some integer $m \geq 1$ if

$$
\sum_{0 \leq k \leq m}(-1)^{k}\left(\begin{array}{c}
m \\
k
\end{array}\right) T^{* m-k} T^{m-k}=0,
$$

where $\left(\begin{array}{c}m \\ k\end{array}\right)$ be the binomial coefficient. In [19],J.W.Helton initiated the study of operator $T$ which satisfy the identity (1.1) and in [1], J. Agler and M.Stankus studied operator $T$ which satisfy (1.2). The development of the theory of $m$ self-adjoint operators( and the related classes of $m$-isometries was motivated largely by striking connections with differential equations.

A simple manipulation proves that (1.2) is equivalent to

$$
\sum_{0 \leq k \leq m}(-1)^{k}\left(\begin{array}{c}
m \\
k
\end{array}\right)\left\|T^{m-k} x\right\|^{2}
$$

for all $x \in \mathcal{H}$. Evidently, an 1-self-adjoint operator (resp. a 1-isometric operator) is $m$-self-adjoint (resp. $m$-isometric) for all integers $m \geq 1$. Indeed the class of $m$-self-adjoint operators (resp. $m$-isometric operators) is a generalization of the class of self-adjoint operators ( resp. isometric operators ). Major work on $m$-isometries has been done in a long paper consisting of three parts by Agler and Stankus $([1,2,3])$ and have since then attracted the attention of several other authors (see for example [6], [7], [8], [10], [11], [13], [14], [23]). More recently a generalization of these operators to Banach spaces has been studied in the paper of Botelho [9], Sid Ahmed [22], Bayart [4], Bermudez et al. [5], Hoffmann et al. [20] and P.P. Duggal [15]. The equation (1.3) was used to define $m$-isometries on a Banach space by Sid Ahmed [22] and by Botelho [9]. Bayart [4] has replaced the exponent 2 in $(1.3)$ by an $p \in[1, \infty)$ and was introduced 
the following definition: a bounded linear operator $T: X \longrightarrow X$, on a Banach spaces $X$ is an $(m, p)$-isometry ( $m \geq 1$ integer, $p \geq 1$ real) if

$$
\sum_{0 \leq k \leq m}(-1)^{k}\left(\begin{array}{c}
m \\
k
\end{array}\right)\left\|T^{m-k} x\right\|^{p}=0 \quad(x \in X) .
$$

Hoffman et al. [20] considered the above definition with $p>0$ real and studied the role of the second parameter $p$ and also discussed the case $p=\infty$.

In [24], the authors considered an extension of the notion of partial isometries to $m$-partial isometries. We say that $T \in \mathcal{B}(\mathcal{H})$ is an $m$-partial isometry if $T$ satisfies

$$
T \Delta_{T, m}=T \sum_{k=0}^{m}(-1)^{k}\left(\begin{array}{c}
m \\
k
\end{array}\right) T^{* m-k} T^{m-k}=0,
$$

where $\Delta_{T, m}$ is obtained formally from the binomial expansion of $\Delta_{T, m}=$ $\left(T^{*} T-I\right)^{m}$ by understanding $\left(T^{*} T\right)^{m-k}=T^{* m-k} T^{m-k}$. The case when $m=$ 1 is the partial isometries class. The class of $m$-partial isometries properly contains class of $m$-isometries.

Agler and Stankus proved that if $T$ is an $m$-isometry, then $\Delta_{T, m-1} \geq 0$ (Proposition 1.5, [1]).

In the present paper we will give a generalization of $m$-partial isometries and $m$-isometries to $(m, q)$-partial isometries on Hilbert spaces. More precisely we will study the bounded linear operators $T$ on a complex Hilbert space $\mathcal{H}$ that satisfy the identity

$$
T^{q}\left(T^{* m} T^{m}-\left(\begin{array}{c}
m \\
1
\end{array}\right) T^{* m-1} T^{m-1}+-\ldots .+(-1)^{m} I\right)=0 .
$$

We will define an operator satisfying (1.6) to be an $m$-partial isometry of order $q$ on $\mathcal{H}$. The case when $q=m=1$, represent the partial isometries class. If $T$ is injective and it verifies (1.6) is called an $m$-isometry that is deeply studied by J. Agler and M. Stankus in [1]. If $q=1,(m, 1)$-partial isometry becomes $m$-partial isometry.

The contents of the paper are the following. In Section 1 we set up notation and terminology. Furthermore, we collect some facts about $m$-isometries. In Section 2, we will study some properties of $(m, q)$-partial isometries operators. Exactly we will give conditions under which:

- an operator $T$ is $(m, q)$-partial isometry.

- $(m, q)$-partial isometry operator it becomes $m$-partial isometry. 
- $(m, q)$-partial isometry operator it becomes partial isometry.

- $(m, q)$-partial isometry operator it becomes $(m+1, q)$-partial isometry.

- the product and sum of two $(m, q)$-partial isometries operators are $(m, q)$ partial isometry.

- a power of $(m, q)$-partial isometry is an $(m, q)$-partial isometry.

- $(m, q)$-partial isometry operator has the single valued extension property. In order to answer these questions we will briefly review some basic facts about $m$-isometries.

Definition 1.1. A subspace $\mathcal{M}$ of $\mathcal{H}$ is called

1. invariant for $T$ or $T$-invariant if $T(\mathcal{M}) \subset \mathcal{M}$.

2. a reducing subspace for $T$ if both $\mathcal{M}$ and $\mathcal{M}^{\perp}$ are $T$-invariant or equivalently if $\mathcal{M}$ is invariant for both $T$ and $T^{*}$.

Theorem 1.1. ([1]) Let $T \in \mathcal{B}(\mathcal{H})$ be an $m$-isometry for some $m \geq 1$. Then

$$
T^{* n} T^{n}=\sum_{0 \leq k \leq m-1} n^{(k)} \beta_{k}(T)
$$

where

$$
\beta_{k}(T)=\frac{1}{k !} \sum_{0 \leq j \leq k}(-1)^{k-j}\left(\begin{array}{l}
k \\
j
\end{array}\right) T^{* j} T^{j}
$$

and

$$
n^{(k)}=\left\{\begin{array}{l}
0, \text { if } n=0 \\
0 \text { if } n>0 \text { and } k>n \\
\left(\begin{array}{l}
n \\
k
\end{array}\right) k ! \text { if } n>0 \text { and } k \leq n .
\end{array}\right.
$$

Proposition 1.1. ([4, Theorem 2.2]) and [22, Proposition 2.3]). An $(m, p)$-isometry $T \in \mathcal{B}(X)$ is an $(m+1, p)$-isometry.

Theorem 1.2. ([20], Proposition 2.1) Let $T \in \mathcal{B}(X)$ be an $(m, p)$ isometry such that for all $x \in X$ there exists a real number $C(x)>0$ such that

$$
\left\|T^{n}(x)\right\| \leq C(x) \quad \forall n \in \mathbb{N} .
$$

Then $T$ is an isometry. 


\section{2. $m$-Partial Isometries of Order $q$}

Definition 2.1. An operator $T \in \mathcal{B}(\mathcal{H})$ is said to be an $(m, q)$-partial isometry or $m$-partial isometry of order $q$ if and only if $T$ satisfies the identity

$$
T^{q}\left(T^{* m} T^{m}-\left(\begin{array}{c}
m \\
1
\end{array}\right) T^{* m-1} T^{m-1}+\ldots-\ldots+(-1)^{m} I\right)=0 .
$$

In particular, if $T$ is a $(2, q)$-partial isometry or a $(3, q)$-partial isometry, then it must satisfy the operator equation

$$
T^{q}\left(T^{* 2} T^{2}-2 T^{*} T+I\right)=0
$$

or

$$
T^{q}\left(T^{* 3} T^{3}-3 T^{* 2} T^{2}+3 T^{*} T-I\right)=0
$$

Remark 2.1. 1. (1,1)-partial isometry operator is an partial isometry.

2. ( $m, 1)$-partial isometry operator is an $m$-partial isometry.

3. $(1, q)$-partial isometry is an partial isometry of order $q$ i.e., $T^{q} T^{*} T=T^{q}$.

4. Every $m$-partial isometry is an $(m, q)$-partial isometry.

5. Every $(m, q)$-partial isometry is an $(m, q+1)$-partial isometry.

The following example shows that there exists an operator which is $(m, q)$ partial isometry but not $(m, 1)$-partial isometry.

Example 2.1. Let $T=\left(\begin{array}{lll}0 & 0 & 1 \\ 0 & 0 & 0 \\ 0 & 0 & 0\end{array}\right) \in \mathcal{B}\left(\mathbb{C}^{3}\right)$. A simple computation shows that $T$ is a $(2,2)$-partial isometry but not a $(2,1)$-partial isometry.

Example 2.2. We now consider [ 24, Theorem 4.3]. Let us fix an orthonormal basis $\left(e_{n}\right)_{n \geq 1}$ of $\mathcal{H}$. For a sequence of complex numbers $\left(\omega_{n}\right)_{n \geq 1}$, the associated weighted operator on $\mathcal{H}$ with

$$
T e_{n}=\omega_{n} e_{n+1} \text { for all } n \geq 1 .
$$

Ii is well know that $T$ is bounded operator if and only if the weighted sequence $\left(\omega_{n}\right)_{n \geq 1}$ is bounded.We assume that $T$ is bounded weighted shift operator. 
Since $T e_{n}=\omega_{n} e_{n+1}$ for all $n \geq 1$, we see that $T^{k} e_{n}=\left(\prod_{n \leq j \leq k+n-1} \omega_{j}\right) e_{n+k}$. Consequently

$$
T^{* k} e_{n}=\left\{\begin{array}{l}
0, \text { if } n \leq k \\
\left(\prod_{n-k \leq j \leq n-1} \overline{\omega_{j}}\right) e_{n-k} \text { if } n>k+1
\end{array}\right.
$$

Therefore

$$
T^{* k} T^{k} e_{n}=\left(\prod_{n \leq j \leq k+n-1}\left|\omega_{j}\right|^{2}\right) e_{n}
$$

$T$ is a $(m, q)$-partial isometry if and only if for any integer $n \geq 1$

$$
\left(\prod_{n \leq j \leq q+n-1} \omega_{j}\right)\left((-1)^{m}+\sum_{1 \leq k \leq m}(-1)^{m-k}\left(\begin{array}{c}
m \\
k
\end{array}\right)\left(\prod_{n \leq j \leq k+n-1}\left|\omega_{j}\right|^{2}\right)\right)=0
$$

Remark 2.2. If $T \in \mathcal{B}(\mathcal{H})$ such that $\mathcal{N}\left(T^{q}\right)$ is a reducing subspace for $T$, then

$$
T^{* k} T^{k}\left(\mathcal{N}\left(T^{q}\right)^{\perp}\right) \subset \mathcal{N}\left(T^{q}\right)^{\perp}, k=1,2, \ldots, . .
$$

The following theorem characterizes some $(m, q)$-partial isometries operators

Theorem 2.1. Let $T \in \mathcal{B}(\mathcal{H})$ such that $\mathcal{N}\left(T^{q}\right)$ is a reducing subspace for $T$. Then the following properties are equivalent.

(1) $T$ is an $(m, q)$-partial isometry.

$$
\sum_{0 \leq k \leq m}(-1)^{m}\left(\begin{array}{c}
m \\
k
\end{array}\right)\left\|T^{m-k} T^{* q} x\right\|^{2}=0, \text { for all } x \in \mathcal{H} .
$$

Proof. First, assume that $T$ is an $(m, q)$-partial isometry. We have that for all $x \in \mathcal{H}$

$$
\begin{aligned}
& T^{q} \sum_{0 \leq k \leq m}(-1)^{k}\left(\begin{array}{c}
m \\
k
\end{array}\right) T^{* m-k} T^{m-k} T^{* q} x=0 \\
\Longrightarrow & \left\langle T^{q} \sum_{0 \leq k \leq m}(-1)^{k}\left(\begin{array}{c}
m \\
k
\end{array}\right) T^{* m-k} T^{m-k} T^{* q} x, x\right\rangle=0 \\
\Longrightarrow & \sum_{0 \leq k \leq m}(-1)^{k}\left(\begin{array}{c}
m \\
k
\end{array}\right)\left\|T^{m-k} T^{* q} x\right\|^{2}=0 .
\end{aligned}
$$


Conversely assume that $\sum_{0 \leq k \leq m}(-1)^{m}\left(\begin{array}{c}m \\ k\end{array}\right)\left\|T^{m-k} T^{* q} x\right\|^{2}=0$, for all $x \in \mathcal{H}$. It follows that

$$
\begin{aligned}
& \sum_{0 \leq k \leq m}(-1)^{k}\left(\begin{array}{c}
m \\
k
\end{array}\right)\left\|T^{m-k} T^{* q} x\right\|^{2}=0 \\
\Longrightarrow & \left\langle T^{q} \sum_{0 \leq k \leq m}(-1)^{k}\left(\begin{array}{c}
m \\
k
\end{array}\right) T^{* m-k} T^{m-k} T^{* q} x, x\right\rangle=0, \forall x \in \mathcal{H} \\
\Longrightarrow & T^{q} \sum_{0 \leq k \leq m}(-1)^{k}\left(\begin{array}{c}
m \\
k
\end{array}\right) T^{* m-k} T^{m-k} T^{* q} x=0, \forall x \in \mathcal{H} .
\end{aligned}
$$

We deduce that

$$
T^{q} \sum_{0 \leq k \leq m}(-1)^{k}\left(\begin{array}{c}
m \\
k
\end{array}\right) T^{* m-k} T^{m-k}=0 \text { on } \overline{\mathcal{R}\left(T^{* q}\right)}=\mathcal{N}\left(T^{q}\right)^{\perp}
$$

As $\mathcal{N}\left(T^{q}\right)$ is a reducing subspace, we have

$$
T^{q} \sum_{0 \leq k \leq m}(-1)^{k}\left(\begin{array}{c}
m \\
k
\end{array}\right) T^{* m-k} T^{m-k}=0 \text { on } \mathcal{N}\left(T^{q}\right)
$$

and hence,

$$
T^{q} \sum_{0 \leq k \leq m}(-1)^{k}\left(\begin{array}{c}
m \\
k
\end{array}\right) T^{* m-k} T^{m-k}=0
$$

Corollary 2.1. Let $T \in \mathcal{B}(\mathcal{H})$ such that $\mathcal{N}\left(T^{q}\right)$ is a reducing subspace for $T$, then the following properties are equivalent

1. $T$ is an $(m, q)$-partial isometry.

2. $\left.T\right|_{\mathcal{N}\left(T^{q}\right)^{\perp}}$ is an m-isometry.

In the following theorem we show that by imposing certain conditions on $(m, q)$-partial isometry operator it becomes $m$-partial isometry.

Theorem 2.2. If $T$ is an $(m, q)$-partial isometry such that $\mathcal{N}(T)=\mathcal{N}\left(T^{2}\right)$ then $T$ is an $m$-partial isometry. 
Proof. By the assumption we have that $\mathcal{N}(T)=\mathcal{N}\left(T^{n}\right)$ for all positive integer $n$. Hence

$$
T^{q}\left(\sum_{0 \leq k \leq m}(-1)^{k}\left(\begin{array}{c}
m \\
k
\end{array}\right) T^{* m-k} T^{m-k}\right)=0 .
$$

implies

$$
T\left(\sum_{0 \leq k \leq m}(-1)^{k}\left(\begin{array}{c}
m \\
k
\end{array}\right) T^{* m-k} T^{m-k}\right)=0 .
$$

Proposition 2.1. If $T$ is an $(m, q)$-partial isometry such that $T^{k}$ is an partial isometry for $k=1,2,3, \ldots m-1$ then $T^{m}$ is a partial isometry for $m \geq q$.

Proof. Since $T$ is an $(m, q)$-partial isometry we have

$$
T^{q} \sum_{0 \leq k \leq m}(-1)^{k}\left(\begin{array}{c}
m \\
k
\end{array}\right) T^{* m-k} T^{m-k}=0
$$

Multiplying the above equation from the left by $T^{m}$ we get

$$
T^{q}\left(T^{m} T^{* m} T^{m}+\sum_{1 \leq k \leq m}(-1)^{k}\left(\begin{array}{c}
m \\
k
\end{array}\right) T^{m} T^{* m-k} T^{m-k}\right)=0 .
$$

Since $T^{k}$ is a partial isometry for $k=1, \ldots, m-1$ we deduce that

$$
T^{q}\left(T^{m} T^{* m} T^{m}+\sum_{1 \leq k \leq m}(-1)^{k}\left(\begin{array}{c}
m \\
k
\end{array}\right) T^{m}\right)=0 .
$$

Thus,

$$
T^{q}\left(T^{m} T^{* m} T^{m}-T^{m}\right)=0
$$

or equivalently

$$
\left(T^{* m} T^{m} T^{* m}-T^{* m}\right) T^{* q}=0
$$

Hence,

$$
T^{m} T^{* m} T^{m}-T^{m}=0 \quad \text { on } \overline{\mathcal{R}\left(T^{* q}\right)}=\mathcal{N}\left(T^{q}\right)^{\perp} .
$$


On the other hand, since $m \geq q$,

$$
T^{m} T^{* m} T^{m}-T^{m}=0 \quad \text { on } \mathcal{N}\left(T^{q}\right) .
$$

Proposition 2.2. Let $T \in \mathcal{B}(\mathcal{H})$ be an $(m, q)$-partial isometry such that $T^{k}$ is a partial isometry for $k=2,3, \ldots, m$. Then $T^{m+q}=T^{m+q} T^{*} T$ i.e., $T$ is an $(1, m+q)$-partial isometry.

Proof. Using the fact that $T$ is an $(m, q)$-partial isometry, we get

$$
T^{q}\left(\sum_{0 \leq k \leq m}(-1)^{k}\left(\begin{array}{c}
m \\
k
\end{array}\right) T^{* m-k} T^{m-k}\right)=0 .
$$

Multiplying the above equation from the left by $T^{m}$ we get the identity

$$
\begin{array}{r}
T^{q}\left(\sum_{0 \leq k \leq m-2}(-1)^{k}\left(\begin{array}{c}
m \\
k
\end{array}\right) T^{m} T^{* m-k} T^{m-k}+(-1)^{m-1} m T^{m} T^{*} T+(-1)^{m} T^{m}\right) \\
=0 .
\end{array}
$$

By the assumption we get

$$
T^{q}\left(\sum_{0 \leq k \leq m-2}(-1)^{k}\left(\begin{array}{c}
m \\
k
\end{array}\right) T^{m}+(-1)^{m-1} m T^{m} T^{*} T+(-1)^{m} T^{m}\right)=0 .
$$

A calculation shows that $T^{m+q}\left(I-T^{*} T\right)=0$. Hence, the desired result.

In the following corollary we show that by imposing certain conditions on $(m, q)$-partial isometry operator it becomes partial isometry.

Corollary 2.2. Let $T \in \mathcal{B}(\mathcal{H})$ be an $(m, q)$-partial isometry such that $T^{k}$ is a partial isometry for $k=2,3, \ldots, m$. If $\mathcal{N}(T)=\mathcal{N}\left(T^{2}\right)$, then $T$ is an partial isometry.

Proof. It is a consequence of Proposition 2.2 and the fact that $\mathcal{N}(T)=$ $\mathcal{N}\left(T^{n}\right)$ for all positive integer $n$.

Theorem 2.3. Let $T \in \mathcal{B}(\mathcal{H})$ be an $(m, q)$-partial isometry such that $\mathcal{N}\left(T^{q}\right)$ is a reducing subspace for $T$. Assume that there exists a constant $M>0$ satisfying

$$
\left\|\left.T^{n}\right|_{\mathcal{N}\left(T^{q}\right)^{\perp}}\right\| \leq M, \forall n \in \mathbb{N},
$$

then $T$ is a $(1, q)$ - partial isometry. 
Proof. If $T$ is an $(m, q)$-partial isometry, then $\left.T\right|_{\mathcal{N}\left(T^{q}\right)^{\perp}}$ is an $m$-isometry, and by Theorem 1.2 applied to the operator $\left.T\right|_{\mathcal{N}\left(T^{q}\right) \perp}$ we have that $\left.T\right|_{\mathcal{N}\left(T^{q}\right)^{\perp}}$ is an isometry. In particular Corollary 2.1 gives $T^{q} T^{*} T=T^{q}$.

The following example shows that a $(m, q)$-partial isometry operator need not be a $(m+1, q)$-partial isometry and vice versa.

Example 2.3. Let $T=\left(\begin{array}{ccc}0 & 0 & 1 \\ 0 & 0 & 0 \\ \frac{1}{\sqrt{2}} & \frac{1}{\sqrt{2}} & 0\end{array}\right) \in \mathcal{B}\left(\mathbb{C}^{3}\right)$ and $S=\left(\begin{array}{ccc}0 & 0 & 0 \\ 0 & \sqrt{\frac{1+\sqrt{5}}{2}} & 0 \\ 0 & 1 & 0\end{array}\right) \in \mathcal{B}\left(\mathbb{C}^{3}\right)$ then a direct computation shows that

- $T$ is a $(1, q)$-partial isometry but is not a $(2,1)$-partial isometry.

- $S$ is a $(2, q)$-partial isometry but is not a $(1,1)$-partial isometry.

It is well know that every $m$-self-adjoint( resp. $m$-isometry) operator is $(m+1)$-self-adjoint (resp. $(m+1)$-isometry) operator.

In the following theorem we show that by imposing certain conditions on $(m, q)$-partial isometry operator it becomes $(m+1, q)$-partial isometry.

Theorem 2.4. Let $T \in \mathcal{B}(\mathcal{H})$ be an $(m, q)$-partial isometry such that $\mathcal{N}\left(T^{q}\right)$ is a reducing subspace for $T$. Then $T$ is an $(m+n, q)$-partial isometry for $n=1,2, \ldots$.

Proof. Two proofs for this theorem will be given.

The First Proof. Since $T$ is an $(m, q)$-partial isometry and $T\left(\mathcal{N}\left(T^{q}\right)^{\perp}\right) \subset$ $\mathcal{N}\left(T^{q}\right)^{\perp}$ it follows that $T / \mathcal{N}\left(T^{q}\right)^{\perp}$ is an $m$-isometry. By Proposition 1.1 applied to the operator $T / \mathcal{N}\left(T^{q}\right)^{\perp}$ we obtain that $T / \mathcal{N}\left(T^{q}\right)^{\perp}$ is an $(m+n)$-isometry and hence $T$ is an $(m+n, q)$-partial isometry.

The second Proof. The standard formula $\left(\begin{array}{c}m+1 \\ k\end{array}\right)=\left(\begin{array}{c}m \\ k\end{array}\right)+\left(\begin{array}{c}m \\ k-1\end{array}\right)$ for binomial coefficients gives that

$$
\begin{aligned}
& \sum_{0 \leq k \leq m+1}(-1)^{k}\left(\begin{array}{c}
m+1 \\
k
\end{array}\right)\left\|T^{m+1-k} T^{* q} x\right\|^{2} \\
= & \sum_{0 \leq k \leq m+1}(-1)^{k}\left(\left(\begin{array}{c}
m \\
k
\end{array}\right)+\left(\begin{array}{c}
m \\
k-1
\end{array}\right)\right)\left\|T^{m+1-k} T^{* q} x\right\|^{2} \\
= & \sum_{0 \leq k \leq m}(-1)^{k}\left(\begin{array}{c}
m \\
k
\end{array}\right)\left\|T^{m-k} T T^{* q} x\right\|^{2}-\sum_{0 \leq k \leq m}(-1)^{k}\left(\begin{array}{c}
m \\
k
\end{array}\right)\left\|T^{m-k} T^{* q} x\right\|^{2}
\end{aligned}
$$




$$
=0 \text {. }
$$

The following example shows that Theorem 2.4 is not necessarily true if $\mathcal{N}(T)$ is not reducing subspace for $T$.

Example 2.4. ([24]) Let $T=\left(\begin{array}{ccc}0 & 0 & \frac{1}{\sqrt{2}} \\ 0 & 0 & \frac{1}{\sqrt{2}} \\ 1 & 0 & 0\end{array}\right) \in \mathcal{B}\left(\mathbb{C}^{3}\right)$. We not that $\mathcal{N}(T)$ is not reducing for $T$ and $T$ is a 1-partial isometry but $T$ is not a 2 partial isometry.

Example 2.5. The operator $T=\left(\begin{array}{lll}0 & 1 & 0 \\ 0 & 0 & 1 \\ 1 & 0 & 0\end{array}\right) \in \mathcal{B}\left(\mathbb{C}^{3}\right)$ is a 1-partial isometry and a 2-partial isometry and $\mathcal{N}(T)$ is a reducing subspace for $T$.

Proposition 2.3. Let $T \in \mathcal{B}(\mathcal{H})$ be an $(m, q)$-partial isometry. Then $T$ $(m+1, q)$-partial isometry if and if $T$ is an $m$-isometry on $\mathcal{R}\left(T T^{* q}\right)$.

Proof. First assume that $T$ is an $(m, q)$-partial isometry and an $(m .+1, q)$ partial isometry we have by $(2.1)$

$$
T^{q} \sum_{0 \leq k \leq m}(-1)^{k}\left(\begin{array}{c}
m \\
k
\end{array}\right) T^{* m-k} T^{m-k}=0
$$

and

$$
T^{q} \sum_{0 \leq k \leq m+1}(-1)^{k}\left(\begin{array}{c}
m+1 \\
k
\end{array}\right) T^{* m+1-k} T^{m+1-k}=0
$$

Combining (2.4) and (2.5) we obtain

$$
T^{q} T^{*}\left(\sum_{0 \leq k \leq m}(-1)^{k}\left(\begin{array}{c}
m \\
k
\end{array}\right) T^{* m-k} T^{m-k}\right) T=0 .
$$

Thus implies that

$$
T^{q} T^{*}\left(\sum_{0 \leq k \leq m}(-1)^{k}\left(\begin{array}{c}
m \\
k
\end{array}\right) T^{* m-k} T^{m-k}\right) T T^{* q}=0 .
$$

the above inequality means that we can write

$$
\sum_{0 \leq k \leq m}(-1)^{k}\left(\begin{array}{c}
m \\
k
\end{array}\right)\left\|T^{m-k} T T^{* q} x\right\|^{2}=0, \text { for all } x \in \mathcal{H}
$$


and hence the desired result.

Conversely assume that $T$ is an $(m, q)$-partial isometry and an $m$-isometry on $\mathcal{R}\left(T T^{* q}\right)$. We have that

$$
T^{q} \sum_{0 \leq k \leq m}(-1)^{m-k}\left(\begin{array}{c}
m \\
k
\end{array}\right) T^{* k} T^{k}=0
$$

and

$$
\sum_{0 \leq k \leq m}(-1)^{m-k}\left(\begin{array}{c}
m \\
k
\end{array}\right) T^{* k} T^{k} T T^{* q}=0
$$

The equation (2.7) implies

$$
T^{q} \sum_{0 \leq k \leq m}(-1)^{m-k}\left(\begin{array}{c}
m \\
k
\end{array}\right) T^{* k+1} T^{k+1}=0
$$

or equivalently

$$
T^{q} \sum_{1 \leq k \leq m+1}(-1)^{m-k}\left(\begin{array}{c}
m \\
k-1
\end{array}\right) T^{* k} T^{k}=0 .
$$

Combining (2.6) and (2.9), we obtain

$$
T^{q}\left((-1)^{m} I+\sum_{1 \leq k \leq m}(-1)^{m-k}\left(\left(\begin{array}{c}
m \\
k
\end{array}\right)+\left(\begin{array}{c}
m \\
k-1
\end{array}\right)\right) T^{* k} T^{k}-T^{* m+1} T^{m+1}\right)=0 .
$$

The binomial coefficient identity $\left(\begin{array}{c}m \\ k\end{array}\right)+\left(\begin{array}{c}m \\ k-1\end{array}\right)=\left(\begin{array}{c}m+1 \\ k\end{array}\right)$ for $k=1,2, \ldots m$ gives

$$
T^{q} \sum_{0 \leq k \leq m+1}(-1)^{m+1-k}\left(\begin{array}{c}
m+1 \\
k
\end{array}\right) T^{* k} T^{k}=0 .
$$

This completes the proof.

In [22, Theorem 2.2], it is proved that if $T$ and $S$ commuting bounded linear operators on a Banach space $X$ such that $T$ is a 2-isometry and $S$ is an $m$-isometry, then $S T$ is an $(m+1)$-isometry. This result was improved in [6, Theorem 3.3]: if $T S=S T, T$ is an $(m, q)$-isometry and $S$ is an $(n, q)$-isometry, then $S T$ is an $(m+n-1, q)$-isometry.

It is natural to ask whether the product and sum of two $(m, q)$-partial isometries operators are $(m, q)$-partial isometry. In general they need not be. The following Theorems give an affirmative answer under some conditions. 
Theorem 2.5. Let $T, S \in \mathcal{B}(\mathcal{H})$ are $(m, q)$-partial isometries. The following properties hold:

1. If $S T=T S$ and $\mathcal{R}(S) \subset \mathcal{N}(T)$ or $\mathcal{R}(T) \subset \mathcal{N}(S)$, then $T S$ is an $(m, q)$ partial isometry.

2. If $S T=T S=S^{*} T=T S^{*}=0$, then $T+S$ is an $(m, q)$-partial isometry.

Proof. The proof follows from the Definition 2.1.

Proposition 2.4. Let $T, S \in \mathcal{B}(\mathcal{H})$ such that $T$ is an $(m, q)$-partial isometry and $S$ is an $(n, q)$-partial isometry. The following properties hold:

1. If $S T=T S$ and $\mathcal{R}(S) \subset \mathcal{N}(T)$ or $\mathcal{R}(T) \subset \mathcal{N}(S)$, then $T S$ is an $(m+n, q)$ partial isometry.

2. If $S T=T S=S^{*} T=T S^{*}=0, \mathcal{N}(T)$ is a reducing subspace for $T$ and $\mathcal{N}(S)$ is a reducing subspace for $S$, then $T+S$ is an $(m+n, q)$-partial isometry.

Proof. 1. Clear.

2. Since $T$ is it follows that

$$
\begin{aligned}
& (T S)^{q} \sum_{0 \leq k \leq n+m}(-1)^{n+m-k}\left(\begin{array}{c}
m+n \\
k
\end{array}\right)(T+S)^{* k}(T+S)^{k} \\
= & (T S)^{q} \sum_{0 \leq k \leq n+m}(-1)^{n+m-k}\left(\begin{array}{c}
m+n \\
k
\end{array}\right)\left(T^{* k} T^{k}+S^{* k} S^{k}\right) \\
= & 0 \text { (by Theorem 2.4). }
\end{aligned}
$$

The following example shows that the product of $(m, q)$-partial isometries is not necessarily an $(m, q)$-partial isometry.

Example 2.6. Let $T=\left(\begin{array}{lll}0 & 1 & 0 \\ 0 & 0 & 0 \\ 1 & 0 & 1\end{array}\right)$ and $S=\left(\begin{array}{ccc}0 & 0 & 1 \\ 0 & 1 & 0 \\ \frac{1}{\sqrt{2}} & \frac{1}{\sqrt{2}} & 0\end{array}\right)$ acting on $\mathbb{C}^{3}$. It easy to see that $T$ and $S$ are 1-partial isometries but $T S$ is not a 1-partial isometry.

The following example shows that the sum of $(m, q)$-partial isometries is not necessarily an $(m, q)$-partial isometry. 
Example 2.7. Let $T=\left(\begin{array}{lll}0 & 0 & 0 \\ 0 & 0 & 0 \\ 0 & 0 & 1\end{array}\right)$ and $S=\left(\begin{array}{lll}1 & 0 & 0 \\ 0 & 1 & 0 \\ 0 & 0 & 1\end{array}\right)$ acting on $\mathbb{C}^{3}$. It easy to see that $T$ and $S$ are $(2,2)$-partial isometries but $T+S$ is not a $(2,2)$-partial isometry.

We will use the following remark in the proof of Theorem 2.6.

Remark 2.3. $\quad$ 1. The following characterization of 3-isometry operator follows from the identity (1.7). An operator $T \in \mathcal{B}(\mathcal{H})$ is a 3-isometry if and only if there exist operators $B_{1}\left(T^{*}, T\right)$ and $B_{2}\left(T^{*}, T\right)$ such that for all natural numbers $n$,

$$
T^{* n} T^{n}=I+n B_{1}\left(T^{*}, T\right)+n^{2} B_{2}\left(T^{*}, T\right),
$$

where

$$
B_{1}\left(T^{*}, T\right)=\frac{1}{2}\left(-T^{* 2} T^{2}+4 T^{*} T-3 I\right)
$$

and

$$
B_{2}\left(T^{*}, T\right)=\frac{1}{2}\left(T^{* 2} T^{2}-2 T^{*} T+I\right)
$$

2. From the identity (1.7) the following characterization of 2-isometry holds. For an $T \in \mathcal{B}(\mathcal{H})$, then $T$ is an 2-isometry if and only if

$$
T^{* k} T^{k}=k T^{*} T-(k-1) I, \quad k=1,2, \ldots
$$

Theorem 2.6. Let $T \in \mathcal{B}(\mathcal{H})$ be an $(m, q)$-partial isometry and let $S \in$ $\mathcal{B}(\mathcal{H})$ for which $T S=S T$ and $T S^{*}=S^{*} T$. The following properties hold:

1. if $S$ is an isometry, then $T S$ is an $(m, q)$-partial isometry.

2. if $\mathcal{N}\left(T^{q}\right)$ is a reducing subspace for $T$ and $S$ is an 2-isometry,then $T S$ is an $(m+1, q)$-partial isometry.

3. if $\mathcal{N}\left(T^{q}\right)$ is a reducing subspace for $T$ and $S$ is an 3-isometry, then $T S$ is an $(m+2, q)$-partial isometry.

Proof. $\quad$ 1. Let $x \in \mathcal{H}$, we have

$$
\begin{aligned}
& (T S)^{q} \sum_{0 \leq k \leq m}(-1)^{k}\left(\begin{array}{c}
m \\
k
\end{array}\right)(T S)^{* m-k}(T S)^{m-k}(x) \\
= & (T S)^{q} \sum_{0 \leq k \leq m}(-1)^{k}\left(\begin{array}{c}
m \\
k
\end{array}\right) T^{* m-k} T^{m-k}\left(S^{* m-k} S^{m-k} x\right)
\end{aligned}
$$




$$
\begin{aligned}
& =T^{q} \sum_{0 \leq k \leq m}(-1)^{k}\left(\begin{array}{c}
m \\
k
\end{array}\right) T^{* m-k} T^{m-k}\left(S^{q} x\right) \\
& =0
\end{aligned}
$$

2. From part 2. of Remark 2.4 and Theorem 2.4, it follows that

$$
\begin{aligned}
& (T S)^{q} \sum_{0 \leq k \leq m+1}(-1)^{k}\left(\begin{array}{c}
m+1 \\
k
\end{array}\right)(T S)^{* m+1-k}(T S)^{m+1-k} \\
= & (T S)^{q} \sum_{0 \leq k \leq m+1}(-1)^{k}\left(\begin{array}{c}
m+1 \\
k
\end{array}\right) T^{* m+1-k} T^{m+1-k}\left(S^{* m+1-k} S^{m+1-k}\right) \\
= & (T S)^{q} \sum_{0 \leq k \leq m+1}(-1)^{k}\left(\begin{array}{c}
m+1 \\
k
\end{array}\right) T^{* m+1-k} T^{m+1-k}\left((m+1-k) S^{*} S\right) \\
= & (T S)^{q} \sum_{0 \leq k \leq m+1}(-1)^{k}\left(\begin{array}{c}
m+1 \\
k
\end{array}\right) T^{* m+1-k} T^{m+1-k} k\left(I-S^{*} S\right) \\
= & S^{q} T^{q} \sum_{0 \leq k \leq m}(-1)^{k}\left(\begin{array}{c}
m \\
k
\end{array}\right) T^{* m-k} T^{m-k}\left(I-S^{*} S\right) \\
= & 0 .
\end{aligned}
$$

3. Since $S$ is a 3 -isometry and $T S=S T, T S^{*}=S^{*} T$, we have from equation (2.10) and Theorem 2.4 that

$$
\begin{aligned}
& (S T)^{q} \sum_{0 \leq k \leq m+2}(-1)^{k}\left(\begin{array}{c}
m+2 \\
k
\end{array}\right)(S T)^{* m+2-k}(S T)^{m+2-k} \\
= & S^{q} T^{q} \sum_{0 \leq k \leq m+2}(-1)^{k}\left(\begin{array}{c}
m+2 \\
k
\end{array}\right)(T)^{* m+2-k}(T)^{m+2-k}\left(S^{* m+2-k} S^{m+2-k}\right) \\
= & S^{q} T^{q}\left\{\sum_{0 \leq k \leq m+2}(-1)^{k}\left(\begin{array}{c}
m+2 \\
k
\end{array}\right)(T)^{* m+2-k}(T)^{m+2-k} I+\right. \\
& \sum_{0 \leq k \leq m+2}(-1)^{k}\left(\begin{array}{c}
m+2 \\
k
\end{array}\right)(T)^{* m+2-k}(T)^{m+2-k}(m+2-k) B_{1}\left(S^{*}, S\right)+ \\
& \left.\sum_{0 \leq k \leq m+2}(-1)^{k}\left(\begin{array}{c}
m+2 \\
k
\end{array}\right)(T)^{* m+2-k}(T)^{m+2-k}(m+2-k)^{2} B_{2}\left(S^{*}, S\right)\right\}
\end{aligned}
$$




$$
\begin{gathered}
=\underbrace{S^{q} T^{q} \sum_{1 \leq k \leq m+2}(-1)^{k}\left(\begin{array}{c}
m+2 \\
k
\end{array}\right) k(T)^{* m+2-k}(T)^{m+2-k} A_{m}\left(S^{*}, S\right)}_{I} \\
+\underbrace{S^{q} T^{q} \sum_{k=1}^{m+2}(-1)^{k}\left(\begin{array}{c}
m+2 \\
k
\end{array}\right) k^{2}(T)^{* m+2-k}(T)^{m+2-k}\left(B_{2}\left(S^{*}, S\right)\right)}_{J}
\end{gathered}
$$

where

$$
\begin{aligned}
& A_{m}\left(S^{*}, S\right)=\left(-B_{1}\left(S^{*}, S\right)+(-2(m+2)) B_{2}\left(S^{*}, S\right)\right) \\
& I=S^{q} T^{q} \sum_{1 \leq k \leq m+2}(-1)^{k}(m+2)\left(\begin{array}{c}
m+1 \\
k-1
\end{array}\right)(T)^{* m+2-k}(T)^{m+2-k} A_{m}\left(S^{*}, S\right) \\
& =-(m+2) S^{q} T^{q} \sum_{0 \leq k \leq m+1}(-1)^{k}\left(\begin{array}{c}
m+1 \\
k
\end{array}\right)(T)^{* m+1-k}(T)^{m+1-k} \\
& \left(-B_{1}\left(S^{*}, S\right)+(-2(m+2)) B_{2}\left(S^{*}, S\right)\right) \\
& =0 \text {. } \\
& J=S^{q} T^{q} \sum_{1 \leq k \leq m+2}(-1)^{k}\left(\begin{array}{c}
m+2 \\
k
\end{array}\right) k^{2}(T)^{* m+2-k}(T)^{m+2-k}\left(B_{2}\left(S^{*}, S\right)\right) \\
& =S^{q} T^{q} \sum_{k=1}^{m+2}(-1)^{k}\left(\begin{array}{c}
m+2 \\
k
\end{array}\right)(k(k-1)+k)(T)^{* m+2-k}(T)^{m+2-k}\left(B_{2}\left(S^{*}, S\right)\right) \\
& =S^{q} T^{q} \sum_{1 \leq k \leq m+2}(-1)^{k}\left(\begin{array}{c}
m+2 \\
k
\end{array}\right) k(k-1)(T)^{* m+2-k}(T)^{m+2-k}\left(B_{2}\left(S^{*}, S\right)\right) \\
& =(m+2)(m+1) S^{q} T^{q} \sum_{k=2}^{m+2}(-1)^{k}\left(\begin{array}{c}
m \\
k-2
\end{array}\right)(T)^{* m+2-k}(T)^{m+2-k}\left(B_{2}\left(S^{*}, S\right)\right) \\
& =(m+2)(m+1) S^{q} T^{q} \sum_{0 \leq k \leq m}(-1)^{k}\left(\begin{array}{c}
m \\
k
\end{array}\right)(T)^{* m-k}(T)^{m-k}\left(B_{2}\left(S^{*}, S\right)\right) \\
& =0 \text {. }
\end{aligned}
$$

Hence $I+J=0$. Thus $T S$ is a $(m+2, q)$-partial isometry. 
The following corollary is an immediate consequence of Theorem 2.6.

Corollary 2.3. Let $T \in \mathcal{B}(\mathcal{H})$ be an $(m, q)$-partial isometry and let $S \in \mathcal{B}(\mathcal{H})$ for which $T S=S T$ and $T S^{*}=S^{*} T$. the following properties hold:

1. if $S$ is an isometry, then $T S^{n}$ is an $(m, q)$-partial isometry for all positive integer $n$.

2. if $\mathcal{N}\left(T^{q}\right)$ is a reducing subspace for $T$ and $S$ is an 2-isometry, then $T S^{n}$ is an $(m+1, q)$-partial isometry for all positive integer $n$.

3. if $\mathcal{N}\left(T^{q}\right)$ is a reducing subspace for $T$ and $S$ is an 3-isometry, then $T S^{n}$ is an $(m+2, q)$-partial isometry for all positive integer $n$.

It is clear that if $T$ is an isometry, then $T^{r}$ is also an isometry. Saddi and Sid Ahmed in [24, Theorem 2.1] prove that any power of a $(2,1)$-partial isometry if it has a nontrivial reducing sub space $\mathcal{N}(T)$ is again a $(2,1)$-partial isometry. In [16] it was showed that any power of a $(m, 1)$-partial isometry if it has a nontrivial reducing subspace $\mathcal{N}(T)$ is again a $(m, 1)$-partial isometry. Now we generalize it to $(m, q)$-partial. As the proof is very similar to [24, Theorem 2.1] and ( [16], Theorem 2.17), we omit it.

Theorem 2.7. Let $T \in \mathcal{B}(\mathcal{H})$ be an $(m, q)$-partial isometry such that $\mathcal{N}\left(T^{q}\right)$ is a reducing subspace for $T$. Then any power of $T$ is also an $(m, q)$ partial isometry.

Lemma 2.1. ([21]) Let $n \geq 1$ be an integer, and let $T \in \mathcal{B}(\mathcal{H})$ an operator such that $r(T) \leq 1$. Then the following equality hold

$$
\begin{aligned}
& \sum_{0 \leq k \leq n}\left(\begin{array}{l}
n \\
k
\end{array}\right) \varphi_{\alpha}(T)^{* k} \varphi_{\alpha}(T)^{k} \\
= & \left(1-|\alpha|^{2}\right)^{n}\left(I-\alpha T^{*}\right)^{-n}\left(\sum_{0 \leq k \leq n}(-1)^{k}\left(\begin{array}{l}
n \\
k
\end{array}\right) T^{* k} T^{k}\right)(I-\bar{\alpha} T)^{-n}
\end{aligned}
$$

holds for every conformal automorphism $\varphi_{\alpha}$ of the unit disc of the form $\varphi_{\alpha}(z)=$ $\frac{z-\alpha}{1-\bar{\alpha} z}$ for all $z \in \mathbb{D}$ and $\alpha \in \mathbb{D}$.

Let $\operatorname{Aut}(\mathbb{D})$ be the group of all conformal mapping from $\mathbb{D}$ onto itself ( also called disk automorphisms of $\mathbb{D})$. It is well known that $A u t(\mathbb{D})$ coincides with the set of all Möbius transformations of $\mathbb{D}$ onto itself:

$$
\operatorname{Aut}(\mathbb{D})=\left\{\lambda \varphi_{\alpha}:|\lambda|=1, \alpha \in \mathbb{D}\right\} .
$$


We can now conclude that the conformal automorphisms operate on the class of $m$-isometries.

Corollary 2.4. If $T \in \mathcal{B}(\mathcal{H})$ is an $m$-isometry, then so is $\varphi(T)$ for every $\varphi \in \operatorname{Aut}(\mathbb{D})$.

Proof. It is a consequence of the above lemma.

Put

$$
\begin{aligned}
S_{T^{q}} & :=T^{* q} \Delta_{T^{q}, m-1} T^{q} \\
& =T^{* q}\left(\sum_{0 \leq k \leq m-1}(-1)^{k}\left(\begin{array}{c}
m-1 \\
k
\end{array}\right)\left(T^{* q}\right)^{m-1-k}\left(T^{q}\right)^{m-1-k}\right) T^{q}
\end{aligned}
$$

Proposition 2.5. Let $T \in \mathcal{B}(\mathcal{H})$ be an $(m, q)$-partial isometry such that $\mathcal{N}\left(T^{q}\right)$ is a reducing subspace for $T$, then $S_{T^{q}} \geq 0$ i.e.; $\left\langle S_{T^{q}} x, x\right\rangle \geq 0 \quad \forall x \in \mathcal{H}$.

Proof. For $x \in \mathcal{H}$, we have $\left\langle S_{T^{q}} x, x\right\rangle=\left\langle\Delta_{T^{q}, m-1} T^{q} x, T^{q} x\right\rangle$. According to Corollary 2.1,Proposition 1.1 and ([1] Proposition 1.5) we have $\Delta_{\left.T^{q}\right|_{\mathcal{N}\left(T^{q}\right)^{\perp}}, m-1} \geq$ 0 . Since $T$ reduces $\mathcal{N}\left(T^{q}\right)$ then $T x \in \mathcal{N}\left(T^{q}\right)^{\perp}$ and

$$
\left\langle S_{T^{q}} x, x\right\rangle=\left\langle\Delta_{T^{q}, m-1} T^{q} x, T^{q} x\right\rangle \geq 0 .
$$

Hence the result.

Definition 2.2. An operator $T$ is said to have the single valued extension properties if, for every open subset $\mathcal{U}$ of $\mathbb{C}$, an analytic function $f: \mathcal{U} \longrightarrow \mathcal{H}$ satisfies $(T-\lambda) f(\lambda)=0 \forall \lambda \in \mathcal{U}$, then $f(\lambda)=0 \forall \lambda \in \mathcal{U}$.

Theorem 2.8. ([11]) An $m$-isometric operator $T$ has the single valued extension property.

In the following theorem, we extend this result to some $(m, q)$-partial isometries.

Theorem 2.9. Let $T \in \mathcal{B}(\mathcal{H})$ be an $(m, q)$-partial isometry such that $\mathcal{N}\left(T^{q}\right)$ is a reducing subspace for $T$. Then $T$ has the single valued extension properties.

Proof. Assume that $T$ is $(m, q)$-partial isometry for some positive integer $m$. Let $\lambda \in \mathbb{C}$ and let $\mathcal{U}$ be any open neighborhood of $\lambda$ in $\mathbb{C}$. Assume that $f$ is an analytic $\mathcal{H}$-valued function defined on $\mathcal{U}$ such that

$$
(T-\lambda) f(\lambda) \equiv 0 \text { on } \mathcal{U}
$$


Let $f(\lambda)=f_{1}(\lambda) \oplus f_{2}(\lambda) \in \mathcal{N}\left(T^{q}\right) \oplus \mathcal{N}\left(T^{q}\right)^{\perp}$, then we have

$$
(T-\lambda) f(\lambda) \equiv 0 \Longleftrightarrow(T-\lambda) f_{1}(\lambda)+(T-\lambda) f_{2}(\lambda) \equiv 0 \text { on } \mathcal{U} .
$$

We deduce that $(T-\lambda) T^{q} f_{2}(\lambda)=0$ on $\mathcal{U}$.

Since $T$ is an $m$-isometry on $\mathcal{N}\left(T^{q}\right)^{\perp}$ and hence has the single valued extension property (Theorem 2.8), then $T^{q} f_{2}(\lambda) \equiv 0$ and $f_{2}(\lambda) \equiv 0$. Consequently

$$
(T-\lambda) f(\lambda)=0 \Longleftrightarrow(T-\lambda) f_{1}(\lambda)=0 .
$$

Thus $(T-\lambda) f_{1}(\lambda)=0$ implies that $\lambda^{q} f_{1}(\lambda)=0$ and $f_{1}(\lambda)=0$ if $\lambda \neq 0$. Since $f_{1}(\lambda)=0$ if $\lambda \neq 0$ and $f_{1}$ is analytic, $f_{1} \equiv 0$.

Theorem 2.10. The class of $(m, q)$-partial isometries is closed subset of $\mathcal{B}(\mathcal{H})$ equipped with the uniform operator (norm) topology.

Proof. To see that the class of $(m . q)$-partial isometries is closed, we prove that any strong limit $T \in \mathcal{B}(\mathcal{H})$ of a sequence $\left(T_{p}\right)$ in the class of $\left.(m, q)\right]$-partial isometry also belongs to the class of $(m, q)$-partial isometries, i.e., we let $\left(T_{p}\right)$ be a sequence of operators in the class of $(m . q)$-partial isometries converging to $T \in \mathcal{B}(\mathcal{H})$ in norm:

$$
\left\|T_{p} x-T x\right\| \longrightarrow 0 \text { as } p \longrightarrow \infty \text {, for each } x \in H .
$$

Hence it follows that

$$
\left\|T_{p}^{*} x-T^{*} x\right\|=\left\|\left(T_{p}-T\right)^{*} x\right\| \leq\left\|\left(T_{p}-T\right)^{*}\right\|\|x\|=\left\|T_{p}-T\right\|\|x\| \longrightarrow 0,
$$

whence $\left(T_{p}^{*}\right)$ converges strongly to $T^{*}$.

Since the product of operators is sequentially continuous in the strong topology (see [17], p.62), one concludes that $T_{p}^{q} T_{p}^{* k} T_{p}^{k}$ converge strongly to $T^{q} T^{* k} T^{k}$. Hence the limiting case of (2.1) shows that $T$ belongs to the class of $(m, q)$ partial isometries, completing the proof.

\section{References}

[1] J. Agler and M. Stankus, $m$-Isometric transformations of Hilbert space I, Integral Equations and Operator Theory, 21 (1995), 383-429.

[2] J. Agler, M. Stankus, m-Isometric transformations of Hilbert space II, Integral Equations Operator Theory 23 (1) (1995) 1-48. 
[3] J.Agler, M. Stankus, m-Isometric transformations of Hilbert space III, Integral Equations Operator Theory 24 (4) (1996) 379-421.

[4] F.Bayart, m-isometries on Banach spaces, Math. Nachr. 284 (2011), 2141-2147.

[5] T.Bermúdez, C. Díaz-Mendoza, A.Martinón, Powers of m-isometries, Studia Math. 208 (2012) 249-255.

[6] T.Bermúdez, A.Martinón, J.A.Noda, Products of m-isometries, Linear Algebra and its Applications 438 (2013) 80-86.

[7] T.Bermúdez, A.Martinón, V.Müller, and J. A. Noda, Perturbation of misometries by nilpotent operators, Abstr. Appl. Anal., vol.2014. Article ID 745479, 6 pages.

[8] T.Bermúdez, A.Martinón, and E.Negrín, Weighted shift operators which are m-isometries, Integral Equations Operator Theory 68 (2010), No. 3, 301- 312 .

[9] F.Botelho, On the existence of $n$-isometries on $l_{p^{-}}$spaces. Acta Sci. Math. (Szeged) 76 (2010), No. 1-2, 183-192.

[10] F.Botelho and J. Jamison, Isometric properties of elementary operators, Linear Algebra Appl. 432 (2010), 357-365.

[11] M. Chõ , S.Ôta, K.Tanahashic, A. Uchiyama, Spectral properties of misometric. operators. Functional Analysis, Approximation and Computation.4:2 (2012), 33-39

[12] J. B. Conway, A course in Functional analysis Second Edition. SpringerVerlag 1990.

[13] B.P.Duggal, Tensor product of n-isometries, Linear Alg. Appl. 437(2012), 307-318.

[14] B.P.Duggal, Tensor product of n-isometries II Functional Analysis, Approximation and Computation 4:1(2012), 27-32.

[15] B.P.Duggal, Tensor product of n-isometries III, Functional Analysis, Approximation and Computation 4:2(2012), 61-67.

[16] S.H.Jah, $\quad$ Power of $m$-Partial isometries on Hilbert spaces.Bulletin of Mathematical Analysis and Applications Volume 5 Issue 3 (2013), Pages 79-89. 
[17] P.R. Halmos, A Hilbert Space Problem Book. Springer-Verlag, New York. (1982).

[18] P. R. Halmos and L. J. Wallen, Powers of partial isometries, J. Math. Mech, 19 (1970), 657-663.

[19] J.W.Helton, Operators with a representationby $\mathrm{x}$ on a sobolev spaces.Colloquia Math Soc.J.B.5 (1970).

[20] P. Hoffman, M. Mackey and M. Ó Searcóid, On the second parameter of an $(m ; p)$-isometry, Integral Equation and Operator Theory71(2011), 389-405.

[21] A.Olofsson, An operator-valued Berezin transform and the class of $n$-hypercontractions, Integral Equation and Operator Theory 58 (2007) 503-549.

[22] O.A.Mahmoud Sid Ahmed, $m$-isometric operators on Banach spaces, Asian-European J. Math. 3(2010), 1-19.

[23] S. M. Patel, 2-Isometric Operators, Glasnik Matematicki. Vol. 37(57)(2002), 143-147.

[24] A.Saddi and O. A.Mahmoud Sid Ahmed, $m$-partial isometries on Hilbert spaces Intern .J. Funct. Anal., Operators Theory Appl. 2 (2010), No. 1, 67-83. 
